\title{
Non-covalent Control for Bottom-up Assembly of Functional Supramolecular Wires
}

\author{
Josep Puigmartí-Luis, ${ }^{\dagger}$ Andrea Minoia, ${ }^{\ddagger}$ Hiroshi Uji-i, ${ }^{\S}$ Concepció Rovira, ${ }^{\dagger}$ Jérôme \\ Cornil, ${ }^{\ddagger}$ Steven De Feyter, ${ }^{*}, \S$ Roberto Lazzaroni, ${ }^{*}, \neq$ and David B. Amabilino ${ }^{*}, \dagger$ \\ Institut de Ciència de Materials de Barcelona (CSIC), Campus Universitari, 08193 Bellaterra, \\ Catalonia, Spain, \\ Service de Chimie des Matériaux Nouveaux, Centre de Recherche en Electronique et \\ Photonique Moléculaires, Université de Mons-Hainaut, 20, Place du Parc, B-7000 Mons, \\ Belgium \\ Laboratory of Photochemistry and Spectroscopy, Molecular and Nano Materials, Department \\ of Chemistry, and Institute for Nanoscale Physics and Chemistry, Katholieke Universiteit \\ Leuven (KULeuven), Celestijnenlaan 200-F, 3001 Leuven, Belgium. \\ E-mailamabilino@icmab.es,steven.defeyter@chem.kuleuven.be,Roberto@averell.umh.ac.be
}

\section{Synthesis of Compound 1}

Compound 1 was prepared following the route shown in the scheme below, starting from the known compounds 2 (K.B. Simonsen, N. Svenstrup, J. Lau, O. Simonsen, P. Mørk, G.J. Kristensen, J. Becher, Synthesis, 1996, 407) and 3 (T. Thorsteinsson, M. Másson, K.G. Kristinsson, M.A. Hjálmarsdóttir, H. Hilmarsson, T. Loftsson, J. Med. Chem., 2003, 46, 4173.) following standard procedures which will be detailed elsewhere.

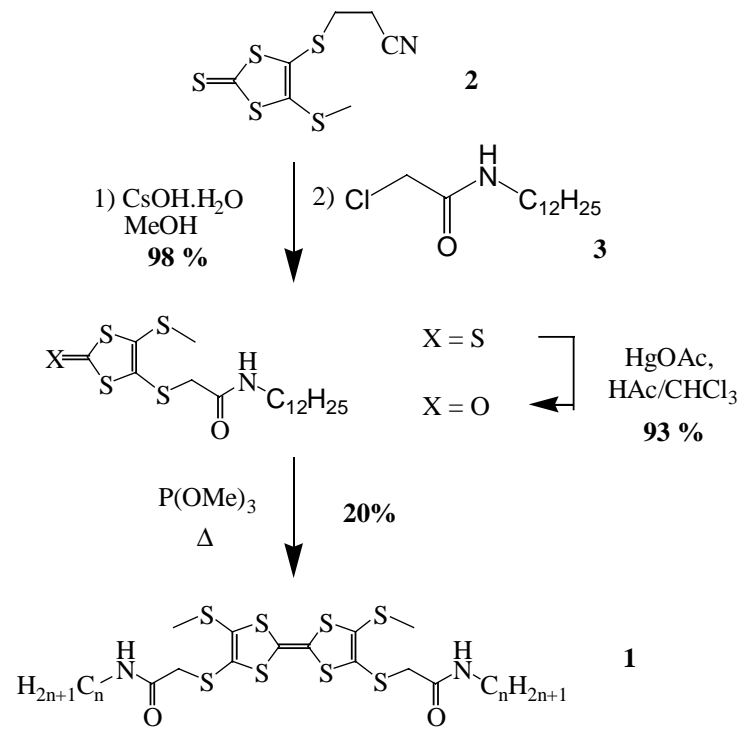

Compound 1 was characterized as follows:

m.p. $83 \stackrel{\circ}{\circ}$ C; LDI-TOF/MS m/z (\%): 811 (M+1, 100); FT-IR; 3300 (s, NH), 2915 (s), 2849 (s), 1646 (s, CONH), 1550 (m, CONH), 1470 (w), 1407 (w), 1377 (w), 1305 (w), 1218 (w, C-N), 1146 (w), 965 (w), $770(w), 718(w, N H) \mathrm{cm}^{-1} ;{ }^{1} \mathrm{H}$ NMR (250 MHz, $\mathrm{CDCl}_{3}$ ): 6,75 (s, 2H, - $\mathrm{SCH}_{2} \mathrm{CONH}-$ ), 3,50 (s, 4H, $-\mathrm{SCH}_{2} \mathrm{CONH}-$ ), 3,28 (q, J=6,2 Hz, 4H, $\left.\mathrm{CONH} \mathrm{CH}{ }_{2}\left(\mathrm{CH}_{2}\right)_{10} \mathrm{CH}_{3}\right), 2,46\left(\mathrm{~s}, 6 \mathrm{H},-\mathrm{SCH}_{3}\right), 1,26\left(\mathrm{~m}, 40 \mathrm{H},-\mathrm{CONH} \mathrm{CH}{ }_{2}\left(\mathrm{CH}_{2}\right){ }_{10} \mathrm{CH}_{3}\right)$, 0,88 (t, $\left.J=6.2 \mathrm{~Hz}, 6 \mathrm{H},-\mathrm{CONH} \mathrm{CH}\left(\mathrm{CH}_{2}\right)_{10} \mathrm{CH}_{3}\right)$ ppm. $\mathrm{C}_{36} \mathrm{H}_{62} \mathrm{~N}_{2} \mathrm{O}_{2} \mathrm{~S}_{8}$ Calc.: C. 53.29: H. 7.70: N. 3.45. Found: C. 53.15: H. 7.80: N. 3.55. 


\section{STM and STS experimental setup}

The compounds were dissolved in 1-octanoic acid, and a drop of the solution was placed on freshly-cleaved highly oriented pyrolitic graphite (HOPG). STM was performed by immersing the tip into the solution until the system formed a tunnel junction and then imaging by scanning at the liquid-solid interface.

The presented STM images were acquired in the variable current mode (constant height) under ambient conditions. In the STM images, white corresponds to the highest and black to the lowest measured tunneling current. STM experiments were performed using a Discoverer scanning tunneling microscope (Topometrix Inc. Santa Barbara, CA) with a typical frame acquisition time of $7 \mathrm{~s}$, along with an external pulse/function generator (model HP $8111 \mathrm{~A}$ ), with negative sample bias. Tips were etched electrochemically from Pt/Ir wire $(80 \% / 20 \%$, diameter $0.2 \mathrm{~mm})$ in a $2 \mathrm{~N}$ $\mathrm{KOH} / 6 \mathrm{~N} \mathrm{NaCN}$ solution in water. The experiments were repeated in several sessions using different tips to check for reproducibility and to avoid artifacts. Note that during the experiments, the STM tip is immersed in the supernatant solution. Different settings for tunneling current and the bias voltage were used, ranging from $0.7 \mathrm{nA}$ to $1 \mathrm{nA}$ and $-0.1 \mathrm{~V}$ to $-1.1 \mathrm{~V}$, respectively. After registration of an STM image of the monolayer structure, the underlying graphite lattice was recorded at the same position by decreasing the bias voltage, serving as an in situ calibration. All STM images contain raw data and are not subjected to any manipulation or image processing. The STS measurements were performed using a Multimode Nanoscope IV (Digital Instruments, Santa Barbara, USA). Current-voltage sweeps were performed with the feedback loop open with the STM tip located over the TTF moieties of 1 located in single fibers in the monolayers and in areas of the layer where the alkyl chains are present. The curves were measured in stable layers when the drift between the tip and the sample was negligible. The averaged unfiltered curve was generated from several measurements done above the TTF moieties in different locations and in different monolayers. Each curve was recorded using anodic and cathodic sweeps, and only those which pass through the set-point (bias voltage, tunneling current) under feedback conditions with an accuracy better than $10 \%$ were used.

\section{Molecular Modelling}

For the molecular dynamics calculations, a stack of four TTF molecules without amide groups was placed onto the surface and physisorbed by minimizing the total energy with a MM force field, providing the starting geometry of the system for the molecular dynamics (MD) simulation. Then a 40ps MD simulation at room temperature in the NVT ensemble with 3D periodic boundary conditions was performed and the state of the system was analyzed along the trajectory. An image of the resulting structure is shown below. 


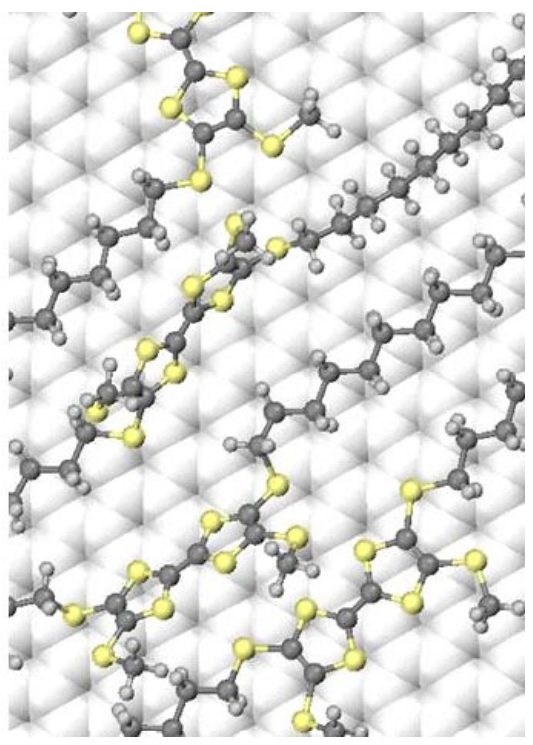

The same procedure was then applied to the stack of the di-amide substituted TTF; the MD trajectory was set to 90ps.

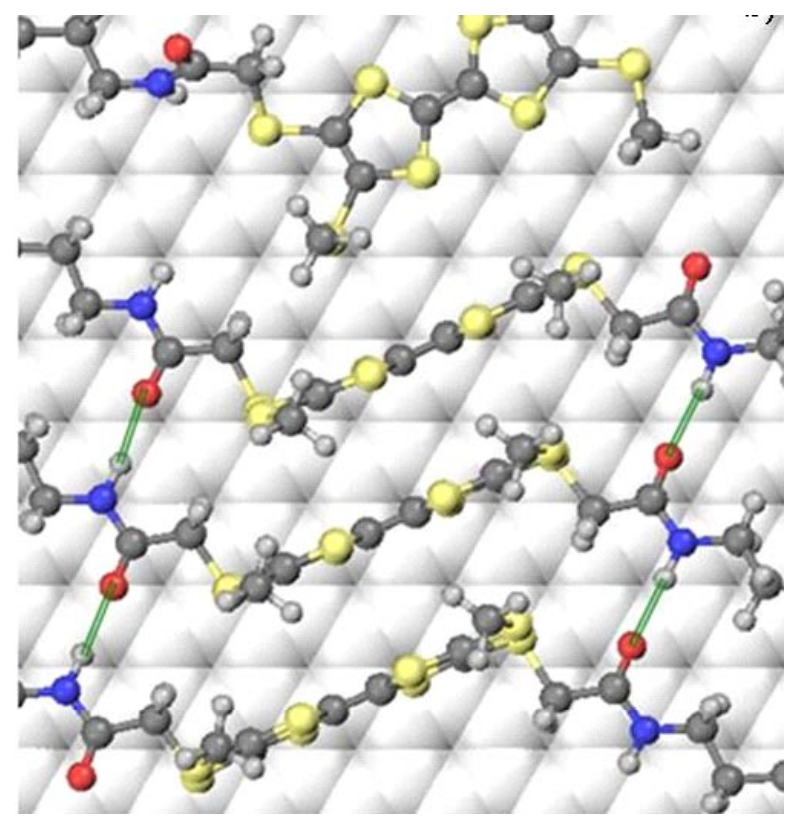

In the quantum-chemical calculations (performed with the Amsterdam Density Functional (ADF) program with the TPZ basis set. Documentation and other information are available at the Web site http://www.scm.com. See also Velde G.T.; Bickelhaupt, F.M.; Baerends, E.J.; Guerra, C.F.; Van Gisbergen, S.J.A.; Snijders, J.G.; Ziegler, T. J. Comp. Chem. 2001, 22, 931-967). The strength of this coupling is reflected for holes and electrons by the amplitude of the transfer integrals

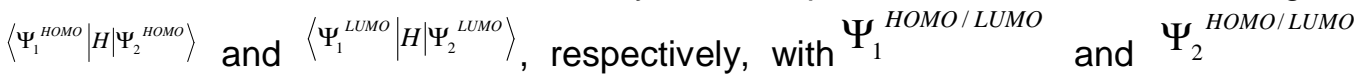
corresponding to the molecular orbitals of the individual molecules (see Senthilkumar, K.; Grozema, F. C.; Bickelhaupt, F. M.; Siebbeles, L. D. A. J. Chem.Phys. 2003, 119, 9809-9817.) The transfer integral for holes and electrons is not calculated here as half the splitting of the HOMO and LUMO levels in the dimer, as traditionally done; it is directly estimated from the evaluation of the matrix 
elements, accounting for the possible energy difference between the HOMO/LUMO levels of the individual molecules in the dimer. Since the electronic coupling strongly depends on the separation and relative orientation of the molecules (Brédas, J.-L.; Calbert, J.-P.; da Silva Filho, D.-A; Cornil, J. PNAS, 2002, 99, 5804-5809.) we have considered the central dimer in the structure shown in the Figure above as the model system for the calculations. 